\title{
TP53 gene rs 1042522 allele G decreases neuroblastoma risk: a two-centre case-control study
}

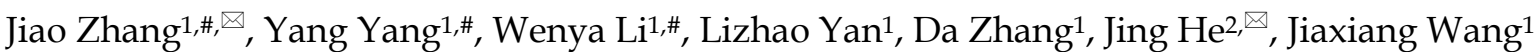 \\ 1. Department of Pediatric Surgery, the First Affiliated Hospital of Zhengzhou University, Zhengzhou 450052, Henan, China \\ 2. Department of Pediatric Surgery, Guangzhou Institute of Pediatrics, Guangzhou Women and Children's Medical Center, Guangzhou Medical University, \\ Guangzhou 510623, Guangdong, China \\ \# These authors contributed equally to this work.
}

$\triangle$ Corresponding authors: Jiao Zhang, Department of Pediatric Surgery, the First Affiliated Hospital of Zhengzhou University, 1 East Jianshe Road, Zhengzhou 450052, Henan, China, Tel./Fax: (+86- 0371) 66279071, e-mail: fcczhangj7@zzu.edu.cn; Jing He, Department of Pediatric Surgery, Guangzhou Institute of Pediatrics, Guangzhou Women and Children's Medical Center, Guangzhou Medical University, 9 Jinsui Road, Guangzhou 510623, Guangdong, China, Tel./Fax: (+86-020) 38076560, e-mail: hejing198374@gmail.com.

(c) Ivyspring International Publisher. This is an open access article distributed under the terms of the Creative Commons Attribution (CC BY-NC) license (https://creativecommons.org/licenses/by-nc/4.0/). See http://ivyspring.com/terms for full terms and conditions.

Received: 2018.05.26; Accepted: 2018.12.08; Published: 2019.01.01

\begin{abstract}
The TP53 gene plays a crucial role in the prevention of cancer formation, which is closely related to TP53 mutation. TP53 gene polymorphism rs 1042522 C>G was largely investigated in various cancers, but its contribution to neuroblastoma is as yet undefined. Here, we evaluated the effect of the TP53 gene rs $1042522 \mathrm{C}>\mathrm{G}$ polymorphism on the development of neuroblastoma in two different regions, with patients from hospitals in both North and South China. The clinical data involved 374 patients and 812 controls. The resulting odds ratios (ORs) and $95 \%$ confidence intervals (Cls) were used with a logistic regression model to determine the intensity of associations between the factors of interest. We found that the TP53 gene rs 1042522 allele G was associated with a reduced risk of developing neuroblastoma. In our stratified analysis of age, sex, primary sites and clinical stages, we observed that male children, older than 18 months, with tumours derived from the mediastinum who had the rs 1042522 CG/GG genotypes were at a decreased risk of developing neuroblastoma. These results indicate that the TP53 gene rs 1042522 allele G may be a potential protective factor against neuroblastoma in Chinese children.
\end{abstract}

Key words: neuroblastoma, TP53, polymorphism, susceptibility

\section{Introduction}

Neuroblastoma is the most common solid tumour in infants. According to statistics, after leukaemia and brain cancer, it has become the third most common cancer in children [1]. Approximately $15 \%$ of children around the world who died of cancer died as a result of neuroblastoma, and approximately $90 \%$ of cases occur in children less than 5 years old [2]. More than half of neuroblastoma patients are diagnosed as high-risk cases with poor prognoses. The current treatment of neuroblastoma is on the rise, and the prognosis for some children with low-risk neuroblastoma has been improved [2]; however, 15\% of children with neuroblastoma die each year. Therefore, looking for more susceptibility genes is an urgent task.
Previous epidemiological studies have shown that there are familial susceptibility genes in the rare familial neuroblastoma [3]. However, in the sporadic cases, these familial susceptibility genes do not play a significant role [4]. Specifically, if parents are exposed to some dangerous environmental contaminant (radiation sources, carcinogens, etc.) before or during pregnancy, there is no evidence that the children of such parents are more likely to be affected by neuroblastoma than the children of unexposed parents. Growing evidence from genome-wide association studies (GWASs) indicate that genetic polymorphisms are implicated in neuroblastoma. For example, the study found that a low risk of neuroblastoma was associated with the genes HSD17B12 and DDX4 [5]. Highly enriched CASC15 
and BARD1, LMO1 polymorphisms have been found in children with high risk of developing neuroblastoma [6-8]. Moreover, candidate gene approaches also identified genetic associations in NEFL [9] and CDKN1B [10] genes with neuroblastoma susceptibility. These studies suggest that genetic polymorphisms may affect neuroblastoma formation in some way.

TP53 gene is located on the short arm of chromosome 17 and is $20 \mathrm{~kb}$ in humans. TP53 plays a role in the inhibition of apoptosis, genomic stability and angiogenesis and has many anti-cancer mechanisms [11]. Among the various SNPs in TP53 gene, rs1042522 G>C is a hotspot polymorphism, which leads to the substitution of proline for arginine (Arg72Pro) at codon 72 of the p53 protein [12]. Multiple data analyses suggest that there are different degrees of association between rs1042522 G>C and susceptibility to cancers, including cervical cancer [13], prostate cancer [14], breast cancer [15], squamous cell carcinoma [16] and lung cancer [17]. However, the role of rs1042522 $\mathrm{C}>\mathrm{G}$ on the risk of neuroblastoma remains un-fully elucidated.

In our previous experiments, we found that the TP53 gene rs1042522 C>G polymorphism may be related to the occurrence of mediastinal neuroblastoma in children with neuroblastoma in South China [18]. To obtain more precise and reliable data on the association between TP53 gene polymorphisms and neuroblastoma risk, we further conducted a more comprehensive case-controls study with participants from two hospitals in North and South China. The current study aims to provide new strategies and ideas for improving the diagnosis and treatment of neuroblastoma.

\section{Materials and methods}

\section{Study subjects}

This study involved two independent case-control populations. The first population was from Henan Province and consisted of 118 neuroblastoma patients and 281 controls [19]. The other population was from our previous study that was conducted in Guangdong Province [18, 20]. All of the children were diagnosed with neuroblastoma, with the diagnosis confirmed by more than two pathologists and their DNA was available for testing. They were all Chinese. All control subjects were free from serious underlying medical conditions and were recruited from the same hospital. Their DNA was also available. The major clinical and biological characteristics of the patient, including age, gender, sites of tumour origin, and the clinical stage of the neuroblastoma (International Neuroblastoma Staging System) were collected. The eligibility criterion for genome-wide genotyping was the ability to obtain 2.0 $\mu \mathrm{g}$ of high quality DNA from peripheral blood. The study was approved by the Institutional Review Committee of the First Affiliated Hospital of Zhengzhou University. The participants in this study gave informed written consent. The demographic characteristics of all participants are shown in Supplemental Table 1. There was no significant difference in age or gender between the case group and the control group in either region. The sites of neuroblastoma origin were also consistent: adrenal glands $(135 ; 36.10 \%)$, retroperitoneal $(109 ; 29.14 \%)$, mediastinal $(87 ; 23.26 \%)$ and neuroblastomas in other regions $(35 ; 9.36 \%)$. Among the samples, 8 sites of origin could not be determined $(2.14 \%)$, and 69 (18.45\%), 96 (25.67\%), 63 (16.84\%), 126 (33.69\%) and 12 $(3.21 \%)$ patients were classified as I, II, III, V and $4 \mathrm{~s}$, respectively, according to INSS criteria, with 8 cases $(2.73 \%)$ classified as NA (not available) due to a lack of information.

\section{SNP selection and genotyping}

The SNP rs1042522 C>G in the TP53 gene was chosen [18]. The genomic DNA was isolated from venous blood samples using the TIANamp Blood DNA Kit (TianGen Biotech Co. Ltd., Beijing, China) according to manufacturer's instructions. Genotyping was performed by TaqMan real-time PCR as published previously $[18,19]$. To ensure the accuracy of genotyping results, a randomly selected $10 \%$ of the samples were genotyped by the DNA sequencing method. The concordance rate for the quality control samples was $100 \%$.

\section{Statistical analysis}

The goodness-of-fit $\chi^{2}$ test was performed to assess if the selected SNP deviated from Hardy-Weinberg equilibrium among controls. The two-sided $\chi^{2}$ test was used to compare demographic variables and genotype frequencies of the cases and controls. ORs and their corresponding 95\% CIs were computed by unconditional logistic regression analyses with or without adjustment for age and gender. The SAS statistical package (version 9.1; SAS Institute, Cary, NC) was used to perform all statistical analyses. All reported $P$ values were two sided, and a $P$ value $<0.05$ was considered statistically significant.

\section{Results}

\section{TP53 gene rs1042522 C>G polymorphism and neuroblastoma risk}

Table 1 shows the genotype frequencies, combination of TP53 gene rs1042522 C>G polymorphism and the relationship with the risk of neuroblastoma in Henan and Guangdong Provinces. 
Our observations agree with Hardy-Weinberg equilibrium conditions $(P=0.919)$ among the combined controls, using good-of-fit $\chi^{2}$ test. In Henan Province, the genotype frequency distribution of the TP53 gene rs1042522 C>G polymorphism was $37.29 \%$ (CC), $49.15 \%$ (CG) and $13.56 \%$ (GG) in the patients and $29.54 \%$ (CC), $53.02 \%$ (CG) and $17.44 \%$ (GG) in the controls. In Guangdong Province, the distribution was $35.55 \%$ (CC), $42.19 \%$ (CG) and $22.27 \%$ (GG) in the patients and $29.25 \%$ (CC), $48.11 \%$ (CG) and $22.64 \%$ (GG) in the controls. In both provinces combined, the distribution was $36.10 \%$ (CC), $44.39 \%$ (CG) and $19.52 \%$ (GG) in the patients and $29.35 \%$ (CC), $49.82 \%$ (CG) and $20.84 \%$ (GG) in the controls. Looking at Henan Province and Guangdong Province individually, we found that rs1042522 C>G was not associated with the risk of neuroblastoma. However, when we further analysed the relationship between the TP53 gene polymorphism and neuroblastoma risk by combining the study populations in Henan and Guangdong Provinces, we found that rs1042522G carriers were associated with a reduced risk of neuroblastoma (CG/GG vs. CC: adjusted odds ratios $(\mathrm{OR})=0.74,95 \%$ confidence interval $(\mathrm{CI})=0.57-0.95, P$ $=0.020)$.

\section{Stratification analysis}

We further explored the association between the rs1042522 C>G polymorphism and neuroblastoma susceptibility by age, sex, tumour origins and clinical stages (Table 2). Compared with the CC genotype, the rs1042522 CG/GG genotypes were associated with a decreased risk of developing neuroblastoma at ages greater than 18 months (adjusted $\mathrm{OR}=0.70,95 \% \mathrm{CI}=$ $0.51-0.96, P=0.027$ ), male (adjusted $\mathrm{OR}=0.66,95 \% \mathrm{CI}$ $=0.47-0.92, P=0.014)$ and for tumours originating from the mediastinum (adjusted OR $=0.57,95 \% \mathrm{CI}=$ $0.38-0.86, P=0.007)$.

Table 1. Association between TP53 rs1042522 C>G polymorphism and neuroblastoma susceptibility

\begin{tabular}{|c|c|c|c|c|c|c|c|}
\hline Genotype & $\begin{array}{l}\text { Cases } \\
\text { n (\%) }\end{array}$ & $\begin{array}{l}\text { Controls } \\
\text { n (\%) }\end{array}$ & $P$ a & $\begin{array}{l}\text { Crude OR } \\
(95 \% \text { CI })\end{array}$ & $P$ & $\begin{array}{l}\text { Adjusted OR } \\
(95 \% \mathrm{CI})^{\mathrm{b}}\end{array}$ & $P^{b}$ \\
\hline \multicolumn{8}{|c|}{ Henan province } \\
\hline $\mathrm{CC}$ & $44(37.29)$ & $83(29.54)$ & & 1.00 & & 1.00 & \\
\hline CG & $58(49.15)$ & $149(53.02)$ & & $0.73(0.46-1.18)$ & 0.203 & $0.71(0.44-1.15)$ & 0.164 \\
\hline GG & $16(13.56)$ & 49 (17.44) & & $0.62(0.31-1.21)$ & 0.158 & $0.60(0.31-1.18)$ & 0.140 \\
\hline Additive & & & 0.276 & $0.77(0.56-1.07)$ & 0.118 & $0.76(0.55-1.05)$ & 0.099 \\
\hline Dominant & $74(62.71)$ & $198(70.46)$ & 0.129 & $0.71(0.45-1.11)$ & 0.130 & $0.68(0.43-1.08)$ & 0.103 \\
\hline Recessive & $102(86.44)$ & $232(82.56)$ & 0.338 & $0.74(0.40-1.37)$ & 0.340 & $0.74(0.40-1.36)$ & 0.334 \\
\hline \multicolumn{8}{|c|}{ Guangdong province } \\
\hline $\mathrm{CC}$ & $91(35.55)$ & $155(29.25)$ & & 1.00 & & 1.00 & \\
\hline CG & $108(42.19)$ & $255(48.11)$ & & $0.72(0.51-1.02)$ & 0.062 & $0.72(0.51-1.02)$ & 0.065 \\
\hline GG & $57(22.27)$ & $120(22.64)$ & & $0.81(0.54-1.22)$ & 0.309 & $0.80(0.53-1.21)$ & 0.290 \\
\hline Additive & & & 0.175 & $0.88(0.72-1.08)$ & 0.229 & $0.88(0.72-1.08)$ & 0.215 \\
\hline Dominant & $165(64.45)$ & $375(70.75)$ & 0.076 & $0.75(0.55-1.03)$ & 0.075 & $0.75(0.55-1.03)$ & 0.074 \\
\hline Recessive & 199 (77.73) & 410 (77.36) & 0.906 & $0.98(0.68-1.40)$ & 0.906 & $0.97(0.68-1.39)$ & 0.860 \\
\hline \multicolumn{8}{|l|}{ Combined } \\
\hline $\mathrm{CC}$ & $135(36.10)$ & 238 (29.35) & & 1.00 & & 1.00 & \\
\hline CG & 166 (44.39) & 404 (49.82) & & $0.72(0.55-0.96)$ & 0.023 & $0.73(0.55-0.96)$ & 0.023 \\
\hline GG & 73 (19.52) & 169 (20.84) & & $0.76(0.54-1.08)$ & 0.123 & $0.76(0.54-1.08)$ & 0.123 \\
\hline Additive & & & 0.064 & $0.85(0.72-1.01)$ & 0.070 & $0.85(0.72-1.01)$ & 0.070 \\
\hline Dominant & $239(63.90)$ & $573(70.65)$ & 0.020 & $0.74(0.57-0.95)$ & 0.020 & $0.74(0.57-0.95)$ & 0.020 \\
\hline Recessive & $303(80.48)$ & 642 (79.16) & 0.600 & $0.92(0.68-1.25)$ & 0.601 & $0.92(0.68-1.25)$ & 0.596 \\
\hline
\end{tabular}

a $\chi^{2}$ test for genotype distributions between neuroblastoma cases and cancer-free controls

${ }^{\mathrm{b}}$ Adjusted for age and gender

Table 2. Stratification analysis for the association between TP53 rs 1042522 C>G polymorphism and neuroblastoma susceptibility

\begin{tabular}{|c|c|c|c|c|c|c|}
\hline Variables & $\mathrm{CC}$ & CG/GG & Crude OR & $P$ & Adjusted OR a & $P$ a \\
\hline & \multicolumn{2}{|c|}{ (Cases/Controls) } & $(95 \% \mathrm{CI})$ & & $(95 \% \mathrm{CI})$ & \\
\hline \multicolumn{7}{|l|}{ Age, month } \\
\hline$\leq 18$ & $41 / 88$ & $83 / 217$ & $0.82(0.52-1.29)$ & 0.389 & $0.82(0.52-1.29)$ & 0.387 \\
\hline$>18$ & $94 / 150$ & $156 / 356$ & $0.70(0.51-0.96)$ & 0.028 & $0.70(0.51-0.96)$ & 0.027 \\
\hline \multicolumn{7}{|l|}{ Gender } \\
\hline Females & $50 / 99$ & $107 / 242$ & $0.88(0.58-1.32)$ & 0.524 & $0.88(0.58-1.32)$ & 0.537 \\
\hline Males & $85 / 139$ & $132 / 331$ & $0.65(0.47-0.91)$ & 0.013 & $0.66(0.47-0.92)$ & 0.014 \\
\hline \multicolumn{7}{|l|}{ Sites of origin } \\
\hline Adrenal gland & $47 / 238$ & $88 / 573$ & $0.78(0.53-1.14)$ & 0.201 & $0.78(0.53-1.15)$ & 0.204 \\
\hline Retroperitoneal & $29 / 238$ & $58 / 573$ & $0.83(0.52-1.33)$ & 0.440 & $0.84(0.52-1.35)$ & 0.468 \\
\hline Mediastinum & $46 / 238$ & $63 / 573$ & $0.57(0.38-0.86)$ & 0.007 & $0.57(0.38-0.86)$ & 0.007 \\
\hline Others & $11 / 238$ & $24 / 573$ & $0.91(0.44-1.88)$ & 0.791 & $0.91(0.44-1.89)$ & 0.798 \\
\hline \multicolumn{7}{|l|}{ Clinical stages } \\
\hline $\mathrm{I}+\mathrm{II}+4 \mathrm{~s}$ & $59 / 238$ & $106 / 573$ & $0.75(0.53-1.06)$ & 0.104 & $0.75(0.53-1.06)$ & 0.105 \\
\hline $\mathrm{III}+\mathrm{IV}$ & $68 / 238$ & $121 / 573$ & $0.74(0.53-1.03)$ & 0.075 & $0.74(0.53-1.04)$ & 0.079 \\
\hline
\end{tabular}

a Adjusted for age and gender, omitting the corresponding stratification factor. 


\section{Discussion}

Compared with the previous study, in this study, we increased the number of samples and conducted a two-centre case-control study in North and South China. In this study, we observed that the TP53 gene rs1042522 allele G was associated with reduced risk of neuroblastoma. In contrast to the previous study, which found that the TP53 gene rs1042522 C>G polymorphism was associated with a decreased risk of neuroblastoma in children in South China that was not statistically significant [18], in this study we collected data from children in representative hospitals in both North and South China, so we believe this result has reference value. To the best of our knowledge, this was the largest study in China of the TP53 gene rs1042522 C>G polymorphism and neuroblastoma risk.

Some studies have shown that genetic variations of tumour suppressor genes or oncogenes can alter the functions of those genes and may contribute to the development of cancer [11]. TP53 has many anticancer functions and plays a crucial role in apoptosis, genomic stability and the inhibition of angiogenesis. The $C$ to $G$ transversion in codon 72 of the TP53 gene has been associated with increased susceptibility to multiple types of cancers. This polymorphism has been shown to impair TP53 activity, including DNA repair, apoptosis, and cycle arrest [21]. Specifically, TP53 harbouring the wild-type codon 72 showed an increased ability to transactivate p21 and to induce stagnation of growth compared to variants, which may be a key step in DNA damage repair [22]. Mutations in TP53 can produce different isoforms that prevent them from having different mechanistic functions in different cells, thereby changing the cancer phenotype from mild to severe [23].

Although growing studies were accessible regarding TP53 gene rs1042522 C>G polymorphism and the risk of cancers, only two of them focus on neuroblastoma. Cattelani et al. released the first report on TP53 gene rs1042522 C>G polymorphism on neuroblastoma risk. They failed to detect significant relationship between the TP53 gene rs1042522 C>G polymorphism and the risk of neuroblastoma in 288 healthy subjects and 286 neuroblastoma patients of European descent [24]. In a three independent case-control cohorts comprising 10290 individuals conducted by Diskin et al., they demonstrated that TP53 rs78378222 and rs35850753 confer to increase risk of neuroblastoma. However, they were unable to observe any association between the TP53 gene rs1042522 C $>$ G variant and overall survival in 1,809 neuroblastoma patients [25].

In this study, we analysed the patient's gender, age, primary sites of tumour origin, and the clinical stage of disease and found that the TP53 gene reduces the risk of developing neuroblastoma in male patients older than 18 months with tumour of mediastinal origins. The role of rs1042522 G allele on the neuroblastoma risk was different from other studies. There are several potential explanations for such conflicting results. First, the same polymorphism might have different role in cancer risk, depending on different ethnicities, regions, and cancer types. Second, such conflicting role might also be the small sample size of all the studies. Third, other risk factors not analysed in the present study may modify TP53 gene SNP to exert its effect. Due to the reasons mentioned above, the conclusions obtained from our study should be interpreted with caution when extrapolated to other ethnic groups. The current study only focuses on genetic analysis. Functional analysis of SNPs is warranted to characterize the described associations [26-29], which would illustrate the underlying mechanisms of how theses SNPs modify neuroblastoma susceptibility. Although the prognosis of those low-risk neuroblastomas can be improved by the current clinical treatment, the survival rate of those children with high-risk and recurrent neuroblastoma is still not optimistic. Approximately $50 \%$ percent of children with a high-risk disease relapse, and the 5-year survival rate in relapsed cases is only $8 \%$ [30]. Therefore, finding an early marker that can predict or screen for neuroblastoma is of great importance.

Individualized treatment based on biomarkers will be a necessary option in future medical therapy [31]. Obviously, we also realized in this study that merely finding the DNA sequence changes in tumour cells is not enough. Additionally, we should understand and predict the pathogenesis of neuroblastoma in a more comprehensive way through the analysis of data from expression profiling, epigenetics, proteomics and host germ cell changes. In this way, we can look for more accurate predictive biomarkers in the future to distinguish between different children with neuroblastoma, so that different children can receive individualized treatment.

In the future, our research will focus on the effects of other common and rare disease susceptibility variants of the identified alleles known to be associated with elevated risk. When a greater number of risk-variant genes are identified, a stronger genetic score can be established in order to better identify and predict patients with neuroblastoma at different sites and stages [32]. We think we should collect more data, including information on environmental factors, in children with neuroblastoma in China because the precision and 
accuracy of the allele risk analysis will increase as the samples continue to be repeated and expanded [4]. In our recent series of studies [18, 19], the accuracy of this assumption has been seen.

We plan to increase the number of cases in China and to conduct a large number of prospective cohort studies through a multi-centre collaborative project. A more comprehensive and meticulous analysis of all the susceptible genes we find in neuroblastoma and the ongoing refinement of neuroblastoma markers of susceptibility are promising for families with children with poor prognoses.

\section{Supplementary Material}

Supplementary table.

http://www.jcancer.org/v10p0467s1.pdf

\section{Acknowledgements}

This study was supported by grants from the National Natural Science Foundation of China (No: 81502187), the Pearl River S\&T Nova Programme of Guangzhou (No: 201710010086), and the State Clinical Key Specialty Construction Project (Paediatric Surgery) 2013 (No: GJLCZD1301).

\section{Competing Interests}

The authors have declared that no competing interest exists.

\section{References}

1. Maris JM, Hogarty MD, Bagatell R, Cohn SL. Neuroblastoma. Lancet. 2007; 369: 2106-20.

2. Newman EA, Nuchtern JG. Recent biologic and genetic advances in neuroblastoma: Implications for diagnostic, risk stratification, and treatment strategies. Semin Pediatr Surg. 2016; 25: 257-64

3. Mosse YP, Laudenslager M, Longo L, Cole KA, Wood A, Attiyeh EF, et al. Identification of ALK as a major familial neuroblastoma predisposition gene. Nature. 2008; 455: 930-5.

4. Bagatell R, Cohn SL. Genetic discoveries and treatment advances in neuroblastoma. Curr Opin Pediatr. 2016; 28: 19-25.

5. Nguyen le B, Diskin SJ, Capasso M, Wang K, Diamond MA, Glessner J, et al. Phenotype restricted genome-wide association study using a gene-centric approach identifies three low-risk neuroblastoma susceptibility Loci. PLoS Genet. 2011; 7: e1002026.

6. Russell MR, Penikis A, Oldridge DA, Alvarez-Dominguez JR, McDaniel L, Diamond M, et al. CASC15-S Is a Tumor Suppressor lncRNA at the $6 \mathrm{p} 22$ Neuroblastoma Susceptibility Locus. Cancer Res. 2015; 75: 3155-66.

7. Capasso M, Devoto M, Hou C, Asgharzadeh S, Glessner JT, Attiyeh EF, et al. Common variations in BARD1 influence susceptibility to high-risk neuroblastoma. Nat Genet. 2009; 41: 718-23.

8. Wang K, Diskin SJ, Zhang H, Attiyeh EF, Winter C, Hou C, et al. Integrative genomics identifies LMO1 as a neuroblastoma oncogene. Nature. 2011; 469: 216-20.

9. Capasso M, Diskin S, Cimmino F, Acierno G, Totaro F, Petrosino G, et al. Common genetic variants in NEFL influence gene expression and neuroblastoma risk. Cancer Res. 2014; 74: 6913-24.

10. Capasso M, McDaniel LD, Cimmino F, Cirino A, Formicola D, Russell MR, et al. The functional variant rs34330 of CDKN1B is associated with risk of neuroblastoma. J Cell Mol Med. 2017; 21: 3224-30.

11. Surget $\mathrm{S}, \mathrm{Khoury}$ MP, Bourdon JC. Uncovering the role of p53 splice variants in human malignancy: a clinical perspective. Onco Targets Ther. 2013; 7: 57-68.

12. Bergamaschi D, Samuels Y, Sullivan A, Zvelebil M, Breyssens H, Bisso A, et al. iASPP preferentially binds p53 proline-rich region and modulates apoptotic function of codon 72-polymorphic p53. Nat Genet. 2006; 38: 1133-41.

13. Chansaenroj J, Theamboonlers A, Junyangdikul P, Swangvaree S, Karalak A, Chinchai T, et al. Polymorphisms in TP53 (rs1042522), p16 (rs11515 and rs3088440) and NQO1 (rs1800566) genes in Thai cervical cancer patients with HPV 16 infection. Asian Pac J Cancer Prev. 2013; 14: 341-6.
14. Fan S, Hao ZY, Zhang M, Liang CZ. Association between the rs1042522 polymorphism in TP53 and prostate cancer risk: An updated meta-analysis. Chronic Dis Transl Med. 2017; 3: 95-104.

15. Ebner F, Schremmer-Danninger E, Rehbock J. The role of TP53 and p21 gene polymorphisms in breast cancer biology in a well specified and characterized German cohort. J Cancer Res Clin Oncol. 2010; 136: 1369-75.

16. Huang YJ, Niu J, Wei S, Yin M, Liu Z, Wang LE, et al. A novel functional DEC1 promoter polymorphism $-249 \mathrm{~T}>\mathrm{C}$ reduces risk of squamous cell carcinoma of the head and neck. Carcinogenesis. 2010; 31: 2082-90.

17. Zheng D, Chen Y, Gao C, Wei Y, Cao G, Lu N, et al. Polymorphisms of p53 and MDM2 genes are associated with severe toxicities in patients with non-small cell lung cancer. Cancer Biol Ther. 2014; 15: 1542-51.

18. He J, Wang F, Zhu J, Zhang Z, Zou Y, Zhang R, et al. The TP53 gene rs1042522 $\mathrm{C}>\mathrm{G}$ polymorphism and neuroblastoma risk in Chinese children. Aging (Albany NY). 2017; 9: 852-9.

19. Zhang J, Lin H, Wang J, He J, Zhang D, Qin P, et al. LMO1 polymorphisms reduce neuroblastoma risk in Chinese children: a two-center case-control study. Oncotarget. 2017; 8: 65620-6.

20. He J, Zou Y, Wang T, Zhang R, Yang T, Zhu J, et al. Genetic Variations of GWAS-Identified Genes and Neuroblastoma Susceptibility: a Replication Study in Southern Chinese Children. Transl Oncol. 2017; 10: 936-41.

21. Siddique M, Sabapathy K. Trp53-dependent DNA-repair is affected by the codon 72 polymorphism. Oncogene. 2006; 25: 3489-500.

22. Altilia S, Santoro A, Malagoli D, Lanzarini C, Ballesteros Alvarez JA, Galazzo $\mathrm{G}$, et al. TP53 codon 72 polymorphism affects accumulation of mtDNA damage in human cells. Aging (Albany NY). 2012; 4: 28-39.

23. Sabapathy K, Lane DP. Therapeutic targeting of p53: all mutants are equal, but some mutants are more equal than others. Nat Rev Clin Oncol. 2018; 15: 13-30.

24. Cattelani S, Ferrari-Amorotti G, Galavotti S, Defferrari R, Tanno B, Cialfi S, et al. The p53 codon 72 Pro/Pro genotype identifies poor-prognosis neuroblastoma patients: correlation with reduced apoptosis and enhanced senescence by the p53-72P isoform. Neoplasia. 2012; 14: 634-43.

25. Diskin SJ, Capasso M, Diamond M, Oldridge DA, Conkrite K, Bosse KR, et al. Rare variants in TP53 and susceptibility to neuroblastoma. J Natl Cancer Inst. 2014; 106: dju047.

26. Gong J, Tian J, Lou J, Wang X, Ke J, Li J, et al. A polymorphic MYC response element in KBTBD11 influences colorectal cancer risk, especially in interaction with an MYC-regulated SNP rs6983267. Ann Oncol. 2018; 29: 632-9.

27. Li J, Chang J, Tian J, Ke J, Zhu Y, Yang Y, et al. A Rare Variant P507L in TPP1 Interrupts TPP1-TIN2 Interaction, Influences Telomere Length, and Confers Colorectal Cancer Risk in Chinese Population. Cancer Epidemiol Biomarkers Prev. 2018; 27: 1029-35.

28. Zou D, Lou J, Ke J, Mei S, Li J, Gong Y, et al. Integrative expression quantitative trait locus-based analysis of colorectal cancer identified a functional polymorphism regulating SLC22A5 expression. Eur J Cancer. 2018; 93: 1-9.

29. Chang J, Zhong R, Tian J, Li J, Zhai K, Ke J, et al. Exome-wide analyses identify low-frequency variant in CYP26B1 and additional coding variants associated with esophageal squamous cell carcinoma. Nat Genet. 2018; 50: 338-43.

30. Pinto NR, Applebaum MA, Volchenboum SL, Matthay KK, London WB, Ambros PF, et al. Advances in Risk Classification and Treatment Strategies for Neuroblastoma. J Clin Oncol. 2015; 33: 3008-17.

31. Roychowdhury S, Chinnaiyan AM. Translating cancer genomes and transcriptomes for precision oncology. CA Cancer J Clin. 2016; 66: 75-88.

32. Brodeur GM, Pritchard J, Berthold F, Carlsen NL, Castel V, Castelberry RP, et al. Revisions of the international criteria for neuroblastoma diagnosis, staging, and response to treatment. J Clin Oncol. 1993; 11: 1466-77. 